\title{
Como Grothendieck simplificou a geometria algébrica
}

\section{How Grothendieck simplified algebraic geometry}

\author{
*Colin McLarty \\ ${ }^{* *}$ Tradução de Norman R. Madarasz
}

\begin{abstract}
Resumo: Alexandre Grothendieck (1922-2014) foi um dos maiores matemáticos do século 20 e um dos mais atípicos. Nascido na Alemanha a um pai anarquista de origem russa, sua infância foi marcada pela militância política dos seus pais, assim passando por revoluções, guerras e sobrevivência. Descoberto por sua precocidade matemática por Henri Cartan, Grothendieck fez seu doutorado sob orientação de Laurent Schwartz e Jean Dieudonné. As principais contribuições dele são na área da topologia e na geometria algébrica, assim como na teoria das categorias. No final dos anos de 1960, ele se dedicou à militância política e ecológica, organizando a revista Survivre durante três anos. Em 1986, publicou um manuscrito autobiográfico de 1000 páginas, Récoltes et semailles, em que ele descreve sua experiência e sua prática da matemática, assim suas contribuições à comunidade matemática francesa. Pouco comentado na filosofia, as implicações dos seus descobrimentos fora mais recentemente discutidas por Alain Badiou na sua "fenômeno-lógica", em Logiques des mondes (2016) e Arkady Plonitsky, Mathgematics, Science and postclassical Theory (1997), pesquisa trata da semelhança entre os aspectos formais da filosofia de Gilles Deleuze e da topologia de Grothendieck.

Palavras-chave: Geometria algébrica. Topologia. Feixe. Esquema. Variabilidade. Teoria das categorias.
\end{abstract}

* Matemático estadunidense. Especialista da teoria das categorias e da obra de Grothendieck. Truman P. Handy Professor, Case Western Reserve University, Ohio, EUA. <colin.mclarty@ case.edu>.

**A presente tradução foi gentilmente concedida pelo autor do artigo para este dossiê. Agradecemos ao Prof. Dr. Colin McLarty, assim como aos Notices from the American Mathematical Society. O artigo foi publicado originalmente em Notices of the MAS, v. 63, n. 2 (March 2016), p. 257-265. http://dx.doi.org/10.1090/noti338 


\begin{abstract}
Philosophers, and not only philosophers of mathematics, have reason to notice what Grothendieck saw in mathematics and especially how close the highest level theory can be to concrete motivations. Plenty of geometers will say that, far from simplifying anything, Grothendieck made algebraic geometry more complicated, abstract, and difficult. And plenty of philosophers suspect mathematicians like abstraction and difficulty for their own sake. But in fact Grothendieck's ideas grew surprisingly directly from concrete geometric problems known to Riemann and Poincaré. They spread because they made Weil's conjectures in arithmetic easier to understand and finally prove. The archetypal post-Grothendieck textbook Algebraic Geometry by Hartshorne is easier to read, and covers more general theorems, and treats more concrete geometric problems, than the previous leading advanced text Foundations of Algebraic Geometry by Weil. This article describes what Grothendieck simplified and how. Grothendieck says the elegant mathematician Jean-Pierre Serre uses the "method of hammer and chisel," seeking swift, powerful tools to cut through hard problems. And he calls Serre's influence indispensable to his own work. But Grothendieck's ideal is the "method of the rising sea," allowing time to develop a general framework to soften a problem before trying to solve it.
\end{abstract}

Keywords: Algebraic geometry. Topology. Sheave. Scheme. Variety. Category theory.

refácio para Filósofos. Filósofos, e não apenas filósofos da mate-
mática, têm razão para perceber o que Alexandre Grothendieck
viu na matemática e especialmente quão perto o mais alto nível
da teoria pode estar de motivações concretas. Muitos geômetras
dirão que, longe de simplificar qualquer coisa, Grothendieck faz da
geometria algo mais complicada, abstrata e difícil. Muitos filósofos
suspeitam que os matemáticos gostam de abstração e de dificuldade
por si só. Mas, na verdade, as ideais de Grothendieck cresceram
surpreendentemente de maneira direta dos problemas concretos
da geometria conhecidos por Riemann e Poincaré. Seus ideais se
propagaram, pois, faziam as conjecturas de Weil na aritmética mais
fácil de entender e finalmente de provar. O arquetípico livro didático
pós-Grothendieck, Algebraic Geometry, por Hartshorne, é mais fácil
de ler, abarca mais teoremas gerais, e trata de um número maior
de problemas geométricos que o texto anterior mais importante,
Foundations of Algebraic Geometry, por Weil. Este artigo descreve o
que Grothendieck simplificou e como. Grothendieck, relata o elegante
matemático Jean-Pierre Serre, usa o "método do martelo e do cinzel",
procurando ferramentas prontas e poderosas para atravessar problemas 
difíceis. Ele considera a influência de Serre indispensável para seu próprio trabalho. Mas o ideal de Grothendieck é o "método do mar crescente", que dando tempo para desenvolver um programa geral que amoleça um problema antes de tentar resolvê-lo. O problema é "submergido e dissolvido por alguma teoria mais ou menos vasta, indo bem além dos resultados inicialmente a serem estabelecidos." (Récoltes et Semailles, p. 555). Este é o método que encontrarás na sua matemática aqui.

\section{Como Grothendieck simplificou a geometria algébrica}

A ideia de esquema é puerilmente simples - tão simples, tão humilde, que ninguém antes de mim sonhava a se humilhar tão baixo... Cresceu sozinho a partir das únicas demandas da simplicidade e da coerência interna.

(GROTHENDIECK, Récoltes et semailles, p. 28 e 32).

A geometria algébrica nunca foi realmente simples. Não era simples antes ou depois que David Hilbert a refundara em sua álgebra, nem quando André Weil a trouxera na sua teoria dos números. Grothendieck tornou ideias chaves mais simples. Seus esquemas dão uma definição mínima basilar do espaço vislumbrado tão cedo que no tempo de Emmy Noether. Sua cohomologia de funtor derivado revela insights que remetam a Bernhard Riemann até uma forma ágil conveniente à cohomologia étale. Para ser claro, a cohomologia étale não era uma simplificação de nada. Era uma ideia radicalmente nova, feita viável por estas simplificações. Grothendieck adquiriu sua herança de um passo removido das fontes originais, principalmente de Jean-Pierre Serre numa busca compartilhada das conjeturas de André Weil. Ambos Weil e Serre tiraram profundo e diretamente da herança inteira. As ideias originais mostram-se tão próximas às ágeis reformulações de Grothendieck.

\section{Generalidade como aspecto superficial}

O gosto famoso de Grothendieck pela generalidade não é suficiente para explicar seus resultados ou sua influência. Raoul Bott o expressou melhor há cinquenta quatro anos quando descreveu a teorema Grothendieck-Riemann-Roch. Riemann-Roch era o padrão da análise por cento e cinquenta anos, para mostrar como a topologia 
de uma superfície Riemann afeta a análise nesta superficie. Os matemáticos, de Richard Dedekind até Weil, generalizaram-na para curvas sobre qualquer campo ao invés de números complexos. Isto faz com que os teoremas da aritmética sigam o raciocino topológico e analítico sobre o campo $F_{p}$ de algoritmos módulo um primo p. Friedrich Hirzebruch generalizou a versaio complexa para funcionar em todas as dimensões.

Grothendieck provou o teorema para todas as dimensões sobre todos os campos, o que já era um feito, e ele foi mais longe ainda numa maneira muito própria. Além de variedades simples, ele a provou para familias convenientemente contínuas de variedades. Portanto:

"Grothendieck generalizara o teorema ao ponto onde não apenas fica mais geralmente aplicável que a versão de Hirzebruch, mas depende de uma prova mais simples e mais natural." [Bott, 1961].

Isso era o primeiro triunfo concreto para sua nova cohomologia e a nascente teoria de esquema. Reconhecendo que muitos matemáticos desconfiam da generalidade, ele escreveu mais tarde:

'Eu prefiro salientar 'unidade' invés de 'generalidade'. Mas para mim, estes são dois aspectos de uma busca. Unidade representa o aspecto profundo, e generalidade o superficial". [Grothendieck, 19851987 , p. PU 25]

\section{Os inícios da cohomologia}

Superfícies com buracos não são apenas um passatempo divertido, mas de suma importância para a teoria das equaçōes. [Atiyah, 1976, p. 293]

O teorema integral de Cauchy afirma que integrar uma forma holomórfica $\omega$ sobre uma fronteira de qualquer região de uma superfície Riemann dá 0 . Para ver a sua importância, contempla duas curvas fechadas $C$ sobre superfícies Riemann que não são fronteiras completas. Cada um cerca um buraco, e cada um tem $\int_{C} \omega$ não igual 0 para algum $\omega$ holomórfico.

Cortar o toro na curva dotada $C_{1}$ em torno do buraco central do toro dá um tubo, e a curva simples $C 1$ pode limitar apenas uma extremidade.
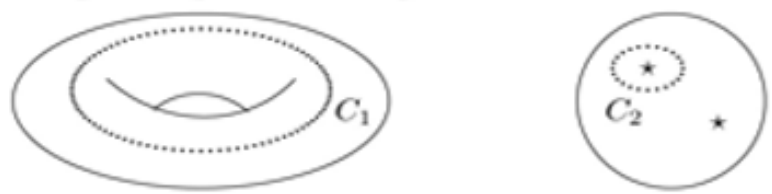

A esfera perfurada no lado direito tem estrelas representando furos, isto é, buracos. As regiōes sobre qualquer lado de $C_{2}$ não são circunscritos nos furos.

Riemann usou este método para calcular integrais. Qualquer curva $C$ e $C^{\prime}$ circundando os mesmos buracos no mesmo número de vezes têm $\int_{C} \omega=\int_{C}, \omega$ para todo $\omega$ holomórfico. Isto é, para que $C$ e o reverso de $C^{\prime}$ formassem o limite completo de um tipo de colar que evita estes buracos. Portanto, $\int_{C} \omega-\int_{C}, \omega=0$.

A cohomologia moderna percebe buracos como obstruções a resolver equações. Dado $\omega$ e um caminho $P:[0,1] \rightarrow S$, seria ótimo calcular o integral $\int_{P} \omega$ ao encontrar uma função $f \operatorname{com~d} f=\omega$, para que $\int_{P} \omega=f\left(P_{1}\right)-f\left(P_{0}\right)$. Nitidamente, não há 
sempre tal função, pois implicaria $\int_{P} \omega=0$ para toda curva fechada $P$. Mas Cauchy, Riemann, e outros perceberem que se $U \subset S$ não cerca nenhum buraco, há funções $f_{U}$ com $\mathrm{d} f_{U}=\omega$ sobre $U$. Buracos são obstruções a colar (patching) soluções locais $f_{U}$ numa solução de $\mathrm{d} f=\omega$ todo sobre $S$.

$\mathrm{O}$ conceito fora generalizado à álgebra e à teoria dos números:

"Ora, agora suponha-se instintivamente que todas as obstruções são melhor descritas em termos de grupos de cohomologia." [SwinnertonDyer, 2001, p. 103]

\section{Grupos de Cohomologia}

"Com homologias, termos compõem de acordo com as regras da adição ordinária." [Poincaré, 1895-1904, pp. 449f.]

Poincaré definiu a adição para curvas para que $C+C^{\prime}$ seja a união de $C$ e $C^{\prime}$, enquanto $-C$ corresponda a reverter a direção de $C$. Portanto, para toda forma $\omega$,

$$
\int_{C+C^{\prime}} \omega=\int_{C} \omega+\int_{C^{\prime}} \omega \text { and } \int_{-C} \omega=-\int_{C} \omega
$$

Quando curvas $C_{1}, \ldots, C_{k}$ formam o limite completo de alguma região, então Poincaré escreve $\Sigma_{k} C_{k} \sim 0$ e fala que a soma é homóloga a 0 . Nestes termos, a teorema integral de Cauchy afirma de maneira concisa:

$$
\Sigma_{k} C_{k} \sim 0 \Rightarrow \int_{\Sigma_{k} C_{k}} \omega=0
$$

para todas as formas holomórficas $\omega$.

Poincaré generalizou esta ideia de homologia para dimensões superiores na base do seu analysis situs, hoje denominado topologia de variedades.

Notadamente, Poincaré publicou duas provas da dualidade Poincaré usando definições diferentes. Sua primeira afirmação dela era falsa. Sua prova misturara incoerências selvagens com insights impressionantes. Para variedades topológicas $M$ de qualquer dimensão $n$ e qualquer $0 \leq i \leq n$, há uma relação estreita entre as subvariedades $i$-dimensionais de $M$ e o $(n-i)$ dimensional. Esta relação é difícil de expressar sem usar a homologia, e Poincaré precisava revisar a sua primeira definição para acertá-la. Mesma a segunda versão dependia de suposições abertamente otimistas acerca de variedades trianguladas.

Os topologistas passaram décadas esclarecendo suas definições e seus teoremas, conseguindo novos resultados no processo. Eles definiram grupos de homologia $\mathrm{H}_{i}(S)$ para cada espaço $S$ e cada dimensão $i \in \mathbb{N}$. Em cada $\mathrm{H}_{1}(S)$, a adição de grupo é o acréscimo de Poincaré de curvas módulo às relações de homologia $\Sigma_{k} C_{k}=0$. Eles também definiram grupos relacionados de cohomologia $H^{i}(S)$ tal que a dualidade de Poincaré afirma que $\mathrm{H}_{i}(M)$ é isomórfica a $\mathrm{H}^{n-i}(M)$ para toda variedade $M n$-dimensional compacto orientável.

As duas definições de homologia se dividem em várias ao usar símplices ou coberturas abertas ou formas diferenciais ou métricas, o que nos leva ao ano 1939:

A topologia algébrica cresce e resolve problemas, mas os não-topologistas estão muito céticos. Na Harvard, Tucker ou talvez Steenrod deram uma aula magistral sobre complexos celulares e a homologia deles, depois da qual um membro distinto do público foi ouvido observando que este tópico alcançava uma complicação algébrica de tal 
nível que não era suscetível de ir mais longe. [Mac Lane, 1976, p. 133]

\section{Coeficientes variáveis e Sequências exatas}

"No seu artigo de Kansas (1955) e no seu artigo de Tôhoku (1957), Grothendieck mostrou que dada uma categoria de feixes a noção de grupos de cohomologia resultava." [Deligne, 1998, p. 16])

A complicação algébrica foi bem mais longe. Métodos em topologia convergiram com métodos da teoria de Galois e conduziram a definir a cohomologia para grupos assim como espaços topológicos. No processo, o que era uma tecnicalidade para Poincaré se tornou central para a cohomologia, a saber, a escolha de coeficientes. Certamente, ele e outros usaram inteiros, números racionais ou reais ou inteiros módulo 2 como coeficientes:

$$
a_{1} C_{1}+\cdots+a_{m} C_{m} \quad a_{i} \in \mathbb{Z} \text {, or } \mathbb{Q} \text {, or } \mathbb{R} \text {, or } \mathbb{Z} / 2 \mathbb{Z} \text {. }
$$

Mas apenas estes tipos de coeficientes relacionados entre si foram usados, pois escolhidos por conveniência para um cálculo dado. Portanto, os topologistas escreverem $\mathrm{H}^{i}(S)$ para o $i$-ro grupo cohomólogo de espaço $S$ e deixaram o grupo coeficiente implícito no contexto.

Em contrapartida, teóricos de grupo escreveram $\mathrm{H}^{i}(G, A)$ para o $i$-ro cohomologia de $G$ com coeficientes em $A$, por que muitos tipos de coeficientes foram usados e eles eram tão interessantes quanto o grupo G. Por exemplo, o famoso Teorema 90 de Hilbert se tornou

$$
\mathrm{H}^{1}\left(\mathrm{Gal}(L / k), L^{\times}\right) \cong\{0\} .
$$

O grupo Galois $\operatorname{Gal}(L / k)$ de uma extensão $L / k$ de campo Galois tem cohomologia trivial de 1-dimensão com coeficientes no grupo multiplicativo $L^{\times}$de todo $0 \neq x \in$ L. Olga Taussky-Todd $[1970$, p. 807$]$ ilustra Teorema 90 ao usá-lo sobre números gaussianos $\mathbb{Q}[i]$ para mostrar que cada triplo Pitagórico de números inteiros tem a forma

$$
m^{2}-n^{2}, 2 m n, m^{2}+n^{2} .
$$

A cohomologia trivial significa que não há obstrução alguma a resolver certos problemas, então o Teorema 90 mostra que certos problemas no campo $L$ têm soluções. Relações algébricas de $\mathrm{H}^{1}\left(\mathrm{Gal}(L / k), L^{\times}\right)$para outros grupos de cohomologia implicam soluções de outros problemas. Bem entendido, Teorema 90 era inventado para resolver muitos problemas décadas antes que o grupo de cohomologia apareceu. Cohomologia organizara e estendera estes usos com tanto sucesso que Emil Artin e John Tate a tornaram basilar à teoria de campo de classe.

Ademais, nos anos de 1940, topologistas adotaram feixes de coeficientes. Um feixe de grupos abelianos $\mathcal{F}$ sobre o espaço $S$ assina grupos abelianos $\mathcal{F}(U)$ a subconjuntos abertos $U \subseteq S$ e homomorfismos $\mathcal{F}(U) \rightarrow \mathcal{F}(V)$ para inclusões de subconjuntos $V \subseteq U$. Portanto, o feixe de funções holomórficas $\mathcal{O}_{M}$ assina o grupo aditivo $\mathcal{O}_{M}(U)$ de funções holomórficas sobre $U$ para cada subconjunto aberto $U \subseteq M$ de uma variedade complexa. Grupos de cohomologia como $\mathrm{H}^{i}\left(M, \mathcal{O}_{M}\right)$ começam a organizar a análise complexa.

Lideres nestes campos consideraram a cohomologia como uma ideia unificada, mas as definições técnicas variam muito. No Séminaire Henri Cartan, palestrantes Cartan, Eilenberg e Serre a organizaram acerca de resoluções. Uma resolução de um 
grupo abeliano $A$ (ou módulo ou feixe) é uma sequência exata de homomorfismos, o que significa que a imagem de cada homomorfismo é o nexo do próximo:

$$
\{0\} \longrightarrow A \longrightarrow I_{0} \longrightarrow I_{1} \longrightarrow \ldots \text {. }
$$

Segue rapidamente que muitas sequências de grupos de cohomologia são também exatas. Esta prova se apoia na Snake Lemma imortalizada por Hollywood numa cena disponível na internet: "Uma prova clara é dada por Jill Clayburgh no início do filme It's My Turn" [Weibel, $1994 \mathrm{p}, 11$ ].

Ao inserir um grupo de cohomologia $\mathrm{H}^{i}(X, \mathcal{F})$ na sequência exata poderia mos$\operatorname{trar} \mathrm{H}^{i}(X, \mathcal{F}) \cong\{0\}$, então as obstruções medidas por $\mathrm{H}^{i}(X, \mathcal{F})$ não existem. Ou poder-se-ia comprovar algum isomorfismo, $\mathrm{H}^{i}(X, \mathcal{F}) \cong \mathrm{H}^{k}(Y, \mathcal{G})$. Portanto, as obstruções medidas por $\mathrm{H}^{i}(X, \mathcal{F})$ correspondem exatamente às mensuradas por $\mathrm{H}^{k}(Y, \mathcal{G})$.

Cohomologia de grupos usa resolução para módulos injetivos $I_{i}$. Um módulo $I$ sobre um anel $R$ é injetivo se, para toda inclusão $R$-módulo $j: N \longmapsto M$, e homomorfismo $f: N \rightarrow I$ existe algum $g: M \rightarrow I$ com $f=g j$. O diagrama é simples:

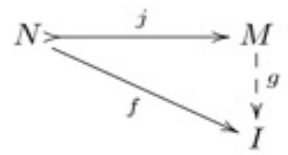

Isto funciona porque cada $R$-módulo $A$ sobre qualquer anel $R$ é embutido em algum $R$-módulo injetivo. Ninguém acreditara algo tão simples poderia funcionar para feixes. A cohomologia para feixes era definida apenas para espaços suficientemente regulares usando vários substitutos topológicos mais complicados para injetivos.

Grothendieck encontrou uma prova inusitada segundo a qual feixes sobre todos os espaços topológicos têm incorporações injetivas. Esta mesma prova funcionara mais tarde para feixes sobre qualquer topologia de Grothendieck.

\section{Tohoku}

Considera o conjunto dos feixes sobre um espaço topológico dado ou, se quiseres, o arsenal prodigioso de todos os "paus métricos" que medem o espaço. Consideres este "conjunto" ou "arsenal" como equipado com sua estrutura mais evidente, de jeito que aparece, para assim falar, "direto na frente do seu nariz"; é isto que denominamos a estrutura de uma "categoria". [Récoltes et sémailles, página 38].

Não definiremos feixes especificamente aqui, tampouco sequências espectrais e outros "desenhos (denominados "diagramas") cheios de flechas que encobrirem o quadro preto", que "escapou totalmente" ao Grothendieck no momento do Seminário Cartan [R\&S, p. 19]. Veremos porque Grothendieck escreveu para Serre em 18 de fevereiro de 1955: "Me livrei do meu horror para sequências espectrais" [Colmez e Serre, 2001, p. 7].

O Seminário Cartan salientou quanto poucas especificidades sobre grupos ou módulos vão nos teoremas básicos. Estes teoremas usam apenas diagramas de homomorfismos. Por exemplo, a soma $A+B$ de grupos abelianos $A, B$ podem ser definidos sozinhos até um isomorfismo pelo fato de que têm homomorfismos $i_{A}: A \rightarrow A+B$ e $i_{B}: B \rightarrow A+B$ e qualquer dois homomorfismos $f: A \rightarrow C$ e 
$g: B \rightarrow C$ dão uma única $u: A+B \rightarrow C$ com $f=u i_{A}$ e $g=u i_{B}$.

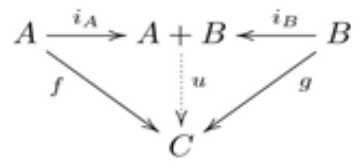

O mesmo diagrama define somas de módulos ou de feixes de grupos abelianos.

Grothendieck [1957, p. 127] pegou as configurações usados pelo Seminário Cartan como seus axiomas de categorias abelianas. Ele acrescentou um axioma adicional AB5 sobre colimites infinitos. Teorema 2.2.2 afirma que se uma categoria Abeliana satisfaz AB5 e mais um axioma da teoria dos conjuntos, então cada objeto nesta categoria está embutido em um objeto injetivo. Estes axiomas, tomados emprestados das categorias modulares, se mantêm também para grupos abelianos sobre qualquer espaço topológico, e a conclusão se aplica.

As pessoas que pensavam que isto era apenas um resultado técnico sobre feixes acharam as ferramentas desproporcionadas em relação ao produto. Elas estavam erradas nos dois casos. Estes axiomas também simplificaram provas de teoremas já conhecidos. Bem especialmente, eles subsumiram muitas sequências espectrais úteis (não todas) sob a sequência espectral de Grothendieck tão simples que pudesse ser o Exercício A.3.50 de [Eisenbud, 2004, p. 683].

As primeiras edições de Algebra de Serge Lang deram aos axiomas de categoria abeliana junto com um exercício famoso: "Pegue qualquer livro sobre álgebra homológica, e prove todos os teoremas sem consultar as provas dadas no livro" [1993, p. 105]. Ele deixou saltar: quando os livros de álgebra homológica começarem a usar provas axiomáticas eles mesmos, mesmo se seus teoremas são afirmados apenas para módulos. David Eisenbud, por exemplo, afirma que suas provas para módulos "generalizam com apenas pouco esforço a [qualquer] categoria abeliana bonita." [2004, p. 620].

Resoluções injetivas em qualquer categoria abeliana dão uma cohomologia de funtor derivado daquela categoria. Era nitidamente geral além de qualquer proporção para os casos então conhecidos. Grothendieck tinha certeza que era a generalidade correta: para uma solução cohomológica a qualquer problema, especialmente às conjecturas de Weil, encontra a correta categoria abeliana.

\section{As conjecturas de Weil}

Esta ideia realmente revolucionária excitou os matemáticos da época, como posso testemunhar de primeira mão. [Serre, 1999, p. 525]

As Conjecturas de Weil que relacionam a aritmética à topologia eram imediatamente reconhecidas como uma grande realização. Weil sabia que concebê-las era o grande momento da sua carreira. Os casos que ele comprovou eram impressionantes. As conjecturas eram lindas demais para não serem verdadeiras, e mesmo assim, eram quase impossíveis a afirmar exatamente.

Weil [1949] apresenta a topologia usando a terminologia do século 19 dos números de Betti. Mas ele era um especialista estabelecido da cohomologia e em conversas:

Naquele tempo, Weil explicava coisas em termos de cohomologia e da formula de ponto fixo de Lefschetz, [mas ele] não queria prever [que funcionaria efetivamente]. De fato, em 1949-50, ninguém pensava que seria possível. [Serre citado em McLarty 2007, p. 305]. 
Lefschetz usou a cohomologia, dependendo da continuidade das variabilidades, para contar os pontos fixos $x=f(x)$ de funções contínuas $f: M \rightarrow M$ sobre variedades. As conjecturas de Weil tratam de espaços definidos sobre campos finitos. Nenhuma versão conhecida destes era contínua. Nem Weil nem ninguém sabia o que poderia funcionar. Grothendieck diz:

Serre explicou as conjecturas de Weil para mim em termos cohomológicos em 1955, e somente nestes termos eles poderiam ter me "fisgado". Ninguém teve a menor ideia de como definir tal cohomologia e eu não tenho certeza que alguém além de Serre e eu, nem Weil se isto for possível, estava profundamente convencido de que tal coisa devesse existir. [R\&S, p. 840]

\section{O que a teoria de esquemas simplificou exatamente}

Kronecker tentava, de fato, descrever e iniciar um novo ramo da matemática, que incluiria ambos a teoria dos números e a geometria algébrica como casos especiais. [Weil, 1952, p. 90].

$\mathrm{O}$ tratamento por Riemann de curvas complexas deixou muito à intuição geométrica. Então, Dedekind e Weber [1882 p. 181] comprovaram um teorema RiemannRoch de uma "perspectiva simples embora rigorosa e plenamente geral" sobre qualquer campo algebricamente fechado $k$ contendo os números racionais. Eles anotaram que $k$ pode ser o campo dos números algébricos. Eles viram que isto dizia respeito à aritmética assim como à análise, e viram bem que o resultado deles era "muito difícil em exposição e expressão. " [1882, p. 235].

As funções meromorfas sobre qualquer superfície compacto Riemann $S$ forma um campo $M(S)$ de grau de transcendência 1 sobre números complexos $\mathbb{C}$. Cada ponto $p \in S$ determina uma função $e_{p}$ de $M(S)$ até $\mathbb{C}+\{\infty\}$. Notavelmente, $e_{p}(f)=f(p)$ quando $f$ é definida em $p_{0}$; e $e_{p}(f)=\infty$ quando $f$ tem um polo em $p$. Então, se ignoramos sumas $\infty+\infty$ :

$$
e_{p}(f+g)=e_{p}(f)+e_{p}(g) \quad e_{p}(f \cdot g)=e_{p}(f) \cdot e_{p}(g) \quad e_{p}\left(\frac{1}{f}\right)=\frac{1}{e_{p}(f)} .
$$

Dedekind e Weber definiram um campo geral de funções algébricas como qualquer extensão $L / K$ de grau de transcendência 1 de campo algébrica fechado $k$. Eles definiram um ponto $p$ de $L$ para ser qualquer função $e_{p}$ de $L$ até $k+\{\infty\}$ que satisfaz estas equações. O teorema Riemann-Roch trata $L$ como se era $M(S)$ para alguma superfície Riemanniana.

Kronecker [1882] realizou alguma "geometria algébrica sobre um campo absolutamente algébrica" [Weil, 1952, p. 92]. Estes campos são as extensões finitas de $\mathbb{Q}$ ou de campos finitos $\mathbb{F}_{p}$. Eles não são fechados algebricamente. Ele visa à "geometria algébrica sobre números inteiros" onde uma variedade poderia ser definida sobre todos estes campos ao mesmo tempo, mas isto era muito difícil naquele momento [Weil, 1952, p. 95].

Os geômetras algébricos italianos dependeram de uma ideia de pontos genéricos de uma variedade complexa $V$, que são pontos complexos ordinários $p \in V$ sem nenhuma propriedade especial aparente. Por exemplo, não é um ponto de singularidade. Noether e Bartel van der Waerden deram pontos genéricos abstratos, que não são pontos complexos, e que não têm propriedades outras senão aquelas que seguem das equações que definem $V$. Van der Waerden [1926] as fez rigorosas mas 
não tão usáveis quanto Weil queria. Oscar Zariski, formado na Itália, trabalhava com Noether em Princeton, e mais tarde com Weil, para dar à geometria algébrica uma base algébrica rigorosa [Parikh, 2009, p. 56].

O corajoso Foundations of Algebraic Geometry de Weil [1946] combinou todos estes métodos no fundamento mais complicado jamais produzido para a geometria algébrica. Para tratar variedades em todas as dimensões sobre campos arbitrária $k$, ele chama extensões $L / k$ algebricamente fechada com grau infinito de transcendência. Além de definir pontos de variedade, ele deve definir subvariedades $V^{\prime} \subseteq V$ em termos de campos de funções racionais. Raynaud [1999] dá um sobrevoo excelente. Listamos três assuntos chave;

(1) Weil tem pontos genéricos. De fato, a variedade definida por polinômios sobre um campo $k$ tem infinitamente muitos pontos genéricos como coordenados transcendentais sobre $k$, todos conjugando entre si por ações Galois sobre $k$.

(2) Weil define variedades algébricas abstratas por dados que lhe dizem como remendar juntas as variedades definidas por equações. Mas estas não existem como espaços simples. Existem somente como conjuntos de variedades concretas mais os dados por remendar.

(3) Weil não poderia definir uma variedade sobre os inteiros embora ele pudesse sistematicamente relatar variedades sobre $\mathbb{Q}$ a outros sobre os campos $\mathbb{F}_{p}$.

\section{Variedades de Serre e feixes coerentes}

Então, Serre [1955] colocou temporariamente pontos genéricos e campos nãofechados de lado para descrever a primeira realmente penetrante cohomologia de variedades algébrica:

Isso se apoia no uso da topologia famosa de Zariski, em que os conjuntos fechados são as subvariedades. O fato impressionante que esta topologia áspera poderia realmente ser colocada a um uso autenticamente matemática era demonstrado por Serre, e produziu uma revolução na linguagem e nas técnicas. [Atiyah, 1966, p. 66]

Digamos que uma variedade ingênua sobre qualquer campo $k$ é um subconjunto $V \subseteq k^{n}$ definido por finitamente muitos polinômios $p_{i}\left(x_{1}, \ldots, x_{n}\right)$ sobre $k$ :

$$
V=\left\{\vec{x} \in k^{n} \mid p_{1}(\vec{x})=\cdots=p_{h}(\vec{x})=0\right\}
$$

Eles formam conjuntos fechados de uma topologia sobre $k^{n}$, chamado a topologia de Zariski. Mesmo as suas interseções infinitas são definidas por finitamente vários polinômios, pois o anel polinomial $k\left[x_{1} \ldots x_{n}\right]$ é Noetheriano. E cada herda a topologia de Zariski onde os conjuntos fechados são as subdivisões $V^{\prime} \subseteq V$ definidas por demais equações.

Estas são topologias ásperas, significando que elas têm poucos subconjuntos abertos ou fechados. Os subconjuntos fechados do campo $k$ mesmo, nesta topologia, são somente subconjuntos finitos mais $k$ inteiro. Portanto, os subconjuntos abertos são o conjunto vazio mais os subconjuntos cofinitos.

Cada variedade ingênua tem uma feixe estrutural $\mathcal{O}_{V}$ que assina a cada Zariski aberto $U \subseteq V$ o anel de funções regulares sobre $U$. Deixando detalhes importantes de fora:

$$
\mathcal{O}_{V}(U)=\left\{\frac{f(\vec{x})}{g(\vec{x})} \text { such that when } \vec{x} \in U \text { then } g(\vec{x}) \neq 0\right\}
$$


Uma variedade de Serre é um espaço topológico $T$ mais um feixe $\mathcal{O}_{T}$ que é localmente isomórfica ao feixe estrutural de uma variedade ingênua. Compara o feixe de funções metamórficas $\mathcal{O}_{M}$ de uma variedade complexa. O aparato do feixe deixa Serre realmente colar variedades juntas sobre remendos compatíveis, como os patches de variedades diferenciáveis são colados juntos. Weil não podia fazer isto com suas variedades abstratas.

Certos feixes relacionados aos feixes estrutural $\mathcal{O}_{T}$ são denominados coerentes. Serre fez deles feixes coeficientes de uma teoria de cohomologia amplamente usada hoje com esquemas. Os laços estreitos de feixes coerentes com feixes estrutural tornam esta cohomologia imprópria para as conjecturas de Weil. Como variedade (ou esquema) $V$ é definido sobre um campo $\mathbb{F}_{p}$ finito, sua cohomologia coerente é definida módulo $p$ e pode contar pontos fixos de mapas $V \rightarrow V$ somente módulo $p$. Mesmo assim,

A principal, e talvez única, inspiração externa para o lançamento repentinamente vigoroso da teoria de esquemas em 1958 era o artigo de Serre conhecido pelo acrônimo FAC. [R\&S, página 28]

\section{Esquemas}

O assunto, grosso modo, era de esvaziar a geometria algébrica das hipóteses parasíticas que a sobrecarregava: campos de base, irredutibilidade, anel finitamente gerado. [Serre, 1989, p. 201]

Esquemas abertamente simplificam a geometria algébrica. Onde os geômetras anteriores complicavam as extensões de campos algebricamente fechados, os teóricos de esquemas usam qualquer anel. Equações polinomiais são substituídas por elementos de anéis. Pontos genéricos se tornam ideais primos. Os conceitos mais intrincados voltam quando precisados, o que é de maneira bastante frequente, mas não é sempre assim e nem desde o início.

De fato, esta perspectiva volta ao trabalho não publicado por Noether, van der Waerden e Wolfgang Krull. Antes de Grothendieck,

a pessoa mais próxima ao pensamento de esquema (no caso affine) era Krull (por volta de 1930). Ele usava sistematicamente o processo de localização, e provou a maioria dos teoremas não-triviais na Álgebra Comutativa. (Serre, e-mail de 21/06/2004, os parênteses de Serre).

Foi o Grothendieck que o fez funcionar. Ele faz de cada anel $R$, o anel de coordenadas de um esquema $\operatorname{Spec}(R)$ denominado espectro de $R$. Os pontos são os ideais primos de $R$, e o esquema tem o feixe estrutural $\mathcal{O}_{R}$ na topologia Zariski para estes pontos, como o feixe estrutural numa variedade de Serre. Segue que os mapas $\operatorname{Spec}(R) \rightarrow \operatorname{Spec}(A)$ correspondem exatamente aos homomorfismos de anel $A \rightarrow R$ em outra direção:

$$
A \stackrel{f}{\longrightarrow} R \quad \operatorname{Spec}(R) \stackrel{\operatorname{Spec}(f)}{\longrightarrow} \operatorname{Spec}(A)
$$

Os pontos podem ser bastante intrincados: "Quando tem que construir uma esquema, geralmente não costuma-se começar com um conjunto de pontos." [Deligne, 1998, p. 12].

Por exemplo, o anel $\mathbb{R}[x]$ de polinômios reais em uma variável é o anel de coordenadas natural para a reta real. Portanto, o espectro $\operatorname{Spec}(\mathbb{R}[x])$ é o esquema 
da reta real. Cada ideal primo não nulo é gerado por um polinômio mônico real irredutível. Estes polinômios são $x-a$ para $a \in \mathbb{R}$, e $x^{2}-2 b x+c$ para $b, c \in \mathbb{R}$ $\operatorname{com} b^{2}<c$. A primeira corresponde aos pontos ordinários $x=a$ da reta real. $\mathrm{O}$ segundo tipo corresponde aos pares das raízes complexas conjugado $b \pm \sqrt{b^{2}-c}$. A esquema $\operatorname{Spec}(\mathbb{R}[x])$ automaticamente inclui ambos pontos reais e complexos, com a nuance que um ponto complexo simples é um par conjugado de raízes complexos.

Uma equação polinomial como $x^{2}+y^{2}=1$ tem muitos tipos de soluções. Poderse-ia pensar de soluções racional e algébrica como tipos de soluções complexas. Mas soluções módulo um primo $p$, tais como $x=2$ e $y=6$ no campo finito $\mathbb{F}_{13}$, não são números complexos. E soluções módulo um primo são diferentes de módulo outro. Todas estas soluções são organizadas em um único esquema

$$
\operatorname{Spec}\left(\mathbb{Z}[x, y] /\left(x^{2}+y^{2}-1\right)\right) .
$$

As funções coordenadas são simplesmente polinomiais inteiros módulo $x^{2}+y^{2}-1$. Os ideais primos não nulo não são simples de modo algum. Eles correspondem às soluções desta equação em todos os campos absolutamente algébricos pelos quais Weil explicava o objetivo de Kronecker, inclusive todos campos finitos. De fato, o mais próximo que Grothendieck chegou a definir os esquemas em Récoltes et Semailles foi quando chamou um esquema de "leque mágico" dobrando juntas as variedades definidas sobre todos estes campos (p. 32). Esta é geometria algébrica sobre os inteiros.

Agora considera o ideal $\left(x^{2}+y^{2}-1\right)$ composto de todos os múltiplos polinomiais de $x^{2}+y^{2}-1$. É primo, portanto é um ponto de $\operatorname{Spec}(\mathbb{Z}[x, y])$. E os esquemas não são espaços Hausdorff. Seus pontos geralmente não são fechados na topologia Zariski. O fechamento deste ponto é $\operatorname{Spec}\left(\mathbb{Z}[x, y] /\left(x^{2}+y^{2}-1\right)\right)$. Este ideal é um ponto genérico da subesquema

$$
\operatorname{Spec}\left(\mathbb{Z}[x, y] /\left(x^{2}+y^{2}-1\right)\right) \longmapsto \operatorname{Spec}(\mathbb{Z}[x, y])
$$

Subesquemas irredutíveis fechados são, para falar irrestritamente, dados por equações no anel coordenado; e cada um tem exatamente um ponto genérico.

No anel $\mathbb{Z}[x, y] /\left(x^{2}+y^{2}-1\right)$, o ideal $\left(x^{2}+y^{2}-1\right)$ aparece como o ideal zero, pois neste anel $x^{2}+y^{2}-1=0$. Portanto, o ideal zero é um ponto genérico para o esquema $\operatorname{Spec}\left(\mathbb{Z}[x, y] /\left(x^{2}+y^{2}-1\right)\right)$ inteiro. O que ocorre neste ponto genérico também ocorre em quase todos os lugares sobre $\operatorname{Spec}\left(\mathbb{Z}[x, y] /\left(x^{2}+y^{2}-1\right)\right)$. Pontos genéricos como estes realizam o que os geômetras algébricos anteriores procuravam nas suas tentativas.

Esquemas justificam intuições mais clássicas também. Os geômetras da Grécia antiga debatiam se a linha de tangente encontra uma curva em algo que é mais que um ponto. A teoria esquemática respondia "sim": uma tangente é um segmento infinitesimal em torno de um ponto.

$\mathrm{O}$ contato da parábola $y=x^{2}$ com o eixo- $x$ em $R^{2}$ é dado simplesmente por $x^{2}=0$. Como variedade, seria somente um espaço ponto $\{0\}$. Mas dá um esquema não trivial $\operatorname{Spec}\left(\mathbb{R}[x] /\left(x^{2}\right)\right)$. As funções coordenadas são polinomiais reais módulo $x^{2}$ ou, em outras palavras, polinomiais lineares reais $a+b x$.

Intuitivamente, $\operatorname{Spec}\left(\mathbb{R}[x] /\left(x^{2}\right)\right)$ é um segmento de linha infinitesimal que contém 0 mas nenhum outro ponto. Este segmento é suficientemente grande para que uma função $a+b x$ sobre ele tenha uma inclinação $b$, mas demasiado pequeno para admitir um derivado segundo. Intuitivamente, um mapa esquema $v \operatorname{de} \operatorname{Spec}\left(\mathbb{R}[x] /\left(x^{2}\right)\right)$ para 
qualquer esquema $S$ é um segmento de uma linha infinitesimal em $S$, isto é, um vetor tangente com ponto base $v(0) \in S$.

$\mathrm{O}$ método de assinatura de Grothendieck, chamado perspectiva relativa, também reflete ideias clássicas. Os geômetras anteriores falariam de, por exemplo, $x^{2}+t \cdot y^{2}=$ 1 como uma equação quadrática em $x, y$ com parâmetro $t$. Mas isso define uma seção cônica $E_{t}$, que é uma elipse ou uma hipérbole ou uma linha dupla que depende de um parâmetro $t$. Mais profundamente, é uma equação cúbica em $x, y, t$ que define uma superfície $E$ que agrega junto todas as curvas $E_{t}$. Sobre os números reais, isto dá um mapa de variedades

$$
E=\left\{\langle x, y, t\rangle \in \mathbb{R}^{3} \mid x^{2}+t \cdot y^{2}=1\right\} \longrightarrow \mathbb{R} \quad\langle x, y, t\rangle \mapsto t
$$

Cada curva $E_{t}$ é uma fibra deste mapa sobre seus parâmetros $t \in \mathbb{R}$. Os geômetras clássicos usavam a continuidade da família de curvas $E_{t}$ agregadas na superfície $E$, mas geralmente eles deixavam a superfície cúbica implícita quando falavam da curva quadrática variável $E_{t}$.

Grothendieck usou meios rigorosos para tratar o mapa esquema $f: X \rightarrow S$ como uma esquema mais simples que o de ambos $X$ e $S .^{2}$ Ele denomina $f$ uma esquema relativo e o trata essencialmente como a fibra única $X_{p} \subset X$, sobre um $p \in S$ indeterminado.

Grothendieck tinha esta perspectiva mesmo antes que ele tivesse esquemas:

Certamente, estamos tão acostumados a colocar algum problema em forma relativa que esquecemos quão revolucionário era naquele momento. A prova que Hirzebruch deu de Riemann-Roch é muito complicada, enquanto a prova da versão relativa, GrothendieckRiemann-Roch, é tão fácil, com o problema sendo transferido ao caso de uma imersão. Isso era fantástico. [Illusie et al., 10’0, p. 1114]

$\mathrm{O}$ que Hirzebruch provou para variedades complexas, Grothendieck provou para mapas convenientes $f: X \rightarrow S$ de variedades sobre qualquer campo $k$. Entre outras vantagens, permitiu a reduzir a prova ao caso de mapas $\mathrm{f}$, denominado imersões, com fibras simples.

Este método depende de mudanças de base que transformam um esquema relativo $f: X \rightarrow S$ sobre uma base $S$ para algum $f^{\prime}: X^{\prime} \rightarrow S^{\prime}$ sobre base $S^{\prime}$. Fibras mesmas são um exemplo. Dada $f: X \rightarrow S$, cada ponto $p \in S$ é definido sobre algum campo $k$, e $p \in S$ equivale a um mapa esquema $p: \operatorname{Spec}(k) \rightarrow S$. A fibra $X_{p}$ faz intuitivamente parte de $X$ estendida sobre $p$, e é precisamente o esquema relativo $X_{p} \rightarrow \operatorname{Spec}(k)$ dado por um pullback:

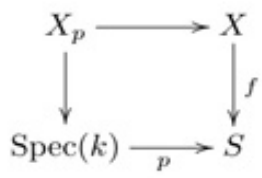

Outros exemplos de mudança de base incluem estender uma esquema $f: Y \rightarrow$ $\operatorname{Spec}(\mathbb{R})$ definido sobre os números reais em um $f^{\prime}: Y^{\prime} \rightarrow \operatorname{Spec}(\mathbb{C})$ sobre números

\footnotetext{
${ }^{2}$ Em 1942, Oscar Zariski provocou algo deste tipo em Weil [Parikh, 2009, p. 70]. Weil levou a ideia mais longe sem transformá-la num método de trabalho. [1952, pp. 91ff].
} 
complexos por pullback sobre o único mapa de esquema de $\operatorname{Spec}(\mathbb{C})$ a $\operatorname{Spec}(\mathbb{R})$ :

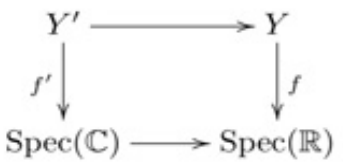

Outras mudanças de base ocorrem nos mapas de esquema $S^{\prime} \rightarrow S$ entre esquemas $S, S^{\prime}$ usados como espaços paramétricos para construções geométricas sérias. Cada uma é simplesmente um pullback no sentido da teoria das categorias. Entretanto, eles codificam informação intrincada e expressam operações que os geômetras anteriores apenas começaram a explorar. Grothendieck e Jean Dieudonné o consideram a maior vantagem da teoria do esquema:

A ideia de "variação" do anel de base que introduzimos recebe uma expressão matemática fácil graças à linguagem functorial, cuja ausência explica sem dúvida a timidez das tentativas anteriores." $[1971$, p. 6]

\section{Cohomologia étale}

No Seminário Chevalley em 21 de abril de 1958, Serre apresentara grupos de cohomologia 1-dimensional novos $\widetilde{\mathrm{H}}^{1}(\mathrm{X}, \underline{\mathrm{G}})$ convenientes para as conjecturas de Weil: "No fim da apresentação oral, Grothendieck falou que isto daria a cohomologia de Weil em todas as dimensões! Achei isto muito otimístico." [Serre, 2001, p. 255]. Naquele setembro, Serre escrevera:

Alguém pode perguntar se for possível definir grupos de cohomologia superiores $\widetilde{\mathrm{H}}^{q}(\mathrm{X}, \underline{\mathrm{G}}) \ldots$ em todas as dimensões. Grothendieck (não publicado) mostrou isto, e parece que quanto $\mathrm{G}$ é finito, estes providenciam a "verdadeira cohomologia" necessária para provar as conjecturas de Weil. Sobre isto, veja a introdução a Grothendieck [1958]. [Serre, 1958, p. 12]

Mais tarde Grothendieck descreveu aquele trabalho não publicado de 1958, dizendo que "duas ideias chaves no lançamento e desenvolvimento da nova geometria eram as de esquema e de topos. Elas aparecerem quase simultaneamente e em simbiose próxima." Especificamente, ele enquadrou "a noção de sítio, a versão técnica, provisional da noção central de topos." [R\&S, páginas P31 e P23n]. Mas antes de prosseguir estas ideias numa cohomologia de dimensão superior, ele usou a ideia de Serre para definir o grupo fundamental de uma variedade ou esquema em analogia próxima com a teoria de Galois.

Percebam que a topologia Zariski registra puncturas muito mais diretamente que registra os buracos como aqueles através do centro do toros ou por dentro do tubo.

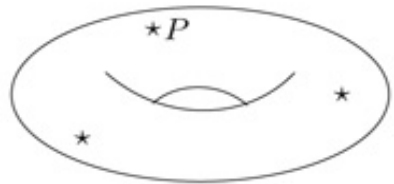

Os subconjuntos fechados de Zariski são (localmente) os conjuntos-nulos de polinômios. Portanto, um subconjunto aberto não-vazio de Zariski do toros é o toros menos um número finito de vários furos (possivelmente nenhum). Tal subconjunto 
pode ou não pode ser perfurado em algum ponto $\mathrm{P}$ ele-mesmo. Assim, os abertos de Zariski eles-mesmos distinguem entre ter e não ter aquele furo. Mas todo subconjunto não-vazio de Zariski cerca o buraco através do centro do toros e aquele através do tubo do toros. Estes subconjuntos por eles-mesmos não podem distinguir entre ter e não ter estes buracos. A cohomologia coerente registra estes buracos ao usar feixes coerentes, que não podem funcionar para as conjecturas de Weil, como notado acima.

Portanto, Serre usou capas com muitas folhas. Considera duas capas de folhas de um toros $T$. Deixa toros $T^{\prime}$ ser duas vezes o comprimento de $T$, com o mesmo diâmetro de tubo. Dobra $T^{\prime}$ duas vezes em torno de $T$ ao longo do tubo:
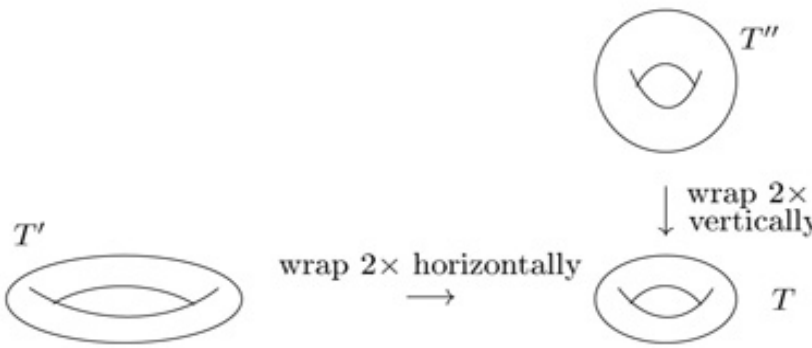

Deixa $T^{\prime \prime}$ ser duas vezes o diâmetro do tubo com o mesmo comprimento de $T$. Dobra-o duas vezes acerca do tubo. A diferença entre estas capas, e ambos de T mesmo, reflete os dois buracos em $\mathrm{T}$.

Riemann criou as superfícies de Riemann como análogas aos campos de números. Como $\mathbb{Q}[\sqrt{2}]$ é uma extensão de campo de grau 2 dos números racionais $\mathbb{Q}$, portanto $T^{\prime} \rightarrow T$ é uma capa de grau 2 de $\mathrm{T}$. Como $\mathbb{Q}[\sqrt{2}] / \mathbb{Q}$ tem um grupo Galois de dois elementos onde um elemento não-idêntico cambia $\sqrt{2} \operatorname{com}-\sqrt{2}$, e $T^{\prime} \rightarrow T$ tem um grupo de simetria de dois elementos sobre $T$ onde a simetria não-idêntica intercambia com as duas folhas de $T^{\prime}$ sobre $T$.

Serre conscientemente estendeu a analogia de Riemann para uma identidade de longo alcance. Ele deu uma definição puramente algébrica de capas não ramificados $S^{\prime} \rightarrow S$ que têm as superfícies supracitadas de Riemann como casos especiais, extensões de campo Galois e mais ainda. Naturalmente, nesta generalidade, alguns teoremas e algumas provas são um pouco técnicos. Mas repetidamente as capas não ramificadas de Serre fazem intuições, tomadas emprestadas das superfícies de Riemann, funcionar para todos estes casos. Grothendieck os usou para dar a primeira teoria útil do grupo fundamental de uma variedade, ou esquema. Eis a homotopia unidimensional. Ele também trabalhou com uma ligeira generalização de capas não ramificadas, denominadas mapas étale, que incluem todas as superfícies de cobrimento algébrico de Riemann.

Serre não calculara a cohomologia de feixes, mas de espaços de fibração isotrivial. Sobre um toros $T$, são espaços aproximadamente mapeados sobre $T$ que podem ser torcidos em torno de $T$, mas podem ser distorcidos por levantamento para um outro toros $T^{\prime \prime \prime} \rightarrow T$ dobrado algumas vezes em torno de cada buraco de $\mathrm{T}$. Enquanto Grothendieck [1955] também usou espaços de fibrações para a cohomologia de umadimensão, ele considerou seus métodos Tôhoku mais promissores para dimensões mais altas. Ele queria alguma noção de feixe que correspondesse às ideias de Serre. 
No ano de 1958, Grothendieck viu que ao invés de definir feixes sobre subconjuntos abertos $U \subseteq S$ de algum espaço $S$, e inclusões $V \subseteq U$, ele poderia usar mapas étale $U \rightarrow S$ para um esquema $S$, e triângulos de mapas étale sobre $S$ :
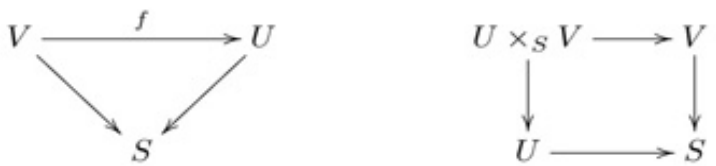

Ele definiu o sítio étale sobre uma esquema $\mathrm{S}$ : ao invés de interseções de subconjuntos abertos $U, V \subseteq S$, usa seus pullbacks $U \times{ }_{S} V$ sobre $S$. Ao invés de cobertores abertos, define uma capa étale de um esquema $S$ como qualquer conjunto de mapas étale $U_{i} \rightarrow S$ tal que a união de todas as imagens é $S$ inteiro. Sítios hoje são frequentemente denominados topologias de Grothendieck, e este sítio pode ser denominado topologia étale sobre $S .^{3}$

Existem duas maneiras de resolver um problema localmente de uma topologia étale sobre $S$. Você pode resolvê-la sobre cada um de um conjunto de subconjuntos abertos de Zariski de $S$ cuja união é $S$, ou você pode resolvê-la usando uma extensão algébrica separável do anel coordenado de $S$. O primeiro dá uma solução atual e global se as soluções locais concordassem em qualquer lugar onde se sobrepõem. A segunda dá uma solução global se uma solução local é um invariante Galoisassim como primeiro faturando um polinômio real sobre os números complexos, e depois mostrando que os fatores são realmente reais. A cohomologia étale pode medir obstruções a colar (patching) tais soluções locais em soluções atuais.

Em 1961, Michael Artin comprovou o primeiro teorema de cohomologia étale de dimensionalidade superior [Artin, 2009, p. 359]. De acordo com David Mumford, este teorema era que o plano com origem deletada tem $\mathrm{H}^{3}$ non-trivial; no contexto de cohomologia étale, significa o plano de coordenado- $x y$, furado na origem, por qualquer campo de coordenados $k$. Portanto, ao menos no caso em que $k$ seja um campo numérico, qualquer cohomologia de Weil deve tratar este plano furado na maneira em que a cohomologia clássica trata o plano coordenado complexo furado no sentido de $\mathbb{C}^{2}-\langle 0,0\rangle$. Isto é topologicamente o espaço real $\mathbb{R}^{4}$ de 4 dimensões furado na sua origem. Tem a cohomologia clássica da esfera-3, $S^{3}$, e isso é não trivial em $\mathrm{H}^{3}$. Assim o resultado de Artin devia se manter em qualquer cohomologia de Weil. Artin provou que se mantém na cohomologia de funtor derivado da categoria de feixes no sítio étale. Hoje, isto é a cohomologia étale.

Artin mostrou que o sítio étale não apenas fornece uma cohomologia de feixe, mas uma que é boa e usável. Os teoremas clássicos da cohomologia sobrevivem com poucas mudanças. Grothendieck convidou Artin para a França para colaborar no seminário que criou Théorie des topos et cohomologie étale [Artin et al., 1972]. O assunto explodiu, mas não iremos mais adiante nele.

Os topos são menos populares hoje na geometria que os esquemas e os sítios. Deligne expressa a sua visão com cautela: "A ferramenta da teoria de topos permitia a construção de cohomologia étale." [1998, p. 15]. Contudo, assim que construída, esta cohomologia é "tão perto da intuição clássica" que, para a maior parte necessita apenas de uma topologia comum mais "um pouco de fé" [1977, p. 5]. Grothendieck "aconselha o leitor no entanto a aprender a linguagem dos topos, pois fornece um princípio unificador extremamente conveniente." [Berthelot et al., 1971, p. VII].

\footnotetext{
${ }^{3}$ Esta perspectiva fora registrada na primavera de 1961 [Grothendieck, 1971, p. 298, s. 4.8].
} 
Terminaremos com a visão de Grothendieck sobre como os esquemas, a sua cohomologia, e os topos convergem todos na cohomologia étale, que de fato nas suas mãos e nas de Deligne forneceram os meios para provar as conjeturas de Weil:

A coisa crucial aqui, do ponto de vista das conjeturas de Weil, é que a nova noção de espaço é suficientemente vasta que pode-se associar a este esquema um "espaço generalizado" ou um "topos" (denominado o "topos étale" do esquema em questão). Certos "invariantes cohomológicos" deste topos (tão infantil que poderia ser!) pareceram ter tido uma boa chance de oferecer "o que precisa" para dar às conjecturas seu pleno significado, e (quem sabe!) porventura fornecer os meios para prová-las." - Récoltes et Semailles, p. P41.

\section{Referências}

ARTIN, Michael. Interview. In Joel Segel, editor, Recountings: Conversations with MIT Mathematicians. A. K. Peters/CRC Press, Wellesley MA, 2009. p. 351-374.

ARTIN, Michael; GROTHENDIECK, Alexander; VERDIER, Jean-Louis. Théorie des Topos et Cohomologie Etale des Schémas. Séminaire de géométrie algébrique du Bois-Marie, 4. Springer-Verlag, 1972. (Três volumes, citado como SGA 4).

ATIYAH, Michael. The role of algebraic topology in mathematics. Journal of the London Mathematical Society, 41 (1966), p. 63-69.

. Bakerian lecture, 1975: Global geometry. Proceedings of the Royal Society of London. Series A, 347, 1650 (1976) p. 291-299.

BERTHELOT, Pierre; GROTHENDIECK, Alexander; ILLUSIE Luc. Théeorie des intersections et théeoréeme de Riemann-Roch. Number 225 in Séminaire de géométrie algébrique du Bois-Marie, 6. Springer-Verlag, 1971. (Geralmente citado como SGA 6).

BOTT, Raoul. Review of A. Borel and J-P. Serre, Le théoréme de Riemann-Roch, in Bull. Soc. Math. France 86, 1958, p. 97-136. Mathematical Reviews (MR0116022 (22\#6817), 1961.

COLMEZ, Pierre; SERRE, Jean-Pierre (ed.). Correspondance Grothen-dieck-Serre. Société Mathématique de France, 2001. Ampliado para Grothen-dieck-Serre Correspondence: Bilingual Edition, American Mathematical Society, and Société Mathématique de France, 2004.

DEDEKIND, Richard; WEBER, Heinrich. Theorie der algebraischen funktionen einer veränderlichen. J. Reine Angew. Math., 92, (1882), p. 181-290. Translated and introduced by John Stillwell as Theory of Algebraic Functions of One Variable, American Mathematical Society and London Mathematical Society, 2012.

DELIGNE, Pierre (ed.). Cohomologie étale, Séminaire de géométrie algébrique du BoisMarie; SGA 4 1/2. Springer-Verlag, 1977. (Geralmente citado como SGA 4 1/2. Este não é estritamente um relatório sobre o Seminário de Grothendieck).

DELIGNE, Pierre. Quelques idées maîtresses de l'oeuvre de A. Grothen-dieck. In Matériaux pour l'Histoire des Mathématiques au XXe Siécle (Nice, 1996), p 11-19. Soc. Math. France, 1998. 
EISENBUD, David. Commutative Algebra. New York: Springer-Verlag, 2004.

GROTHENDIECK, Alexander. A general theory of fibre spaces with structure sheaf. Technical report, University of Kansas, Dept. of Mathematics, 1955.

. Sur quelques points d'algébre homologique. Tôhoku Mathematical Journal, 9 (1957), p. 119-221.

. The cohomology theory of abstract algebraic varieties. In: Proceedings of the International Congress of Mathematicians, 1958. Cambridge University Press, 1958. p. 103-118.

. Revêtements Étales et Groupe Fondamental. Séminaire de géométrie algébrique du Bois-Marie, 1. Springer-Verlag, 1971. (Geralmente citado como SGA 1).

. Récoltes et Semailles\}. Université des Sciences et Techniques du Languedoc, Montpellier, 1985-1987. Publicado em vários volumes sucessivos.

GROTHENDIECK, Alexander; DIEUDONNÉ. Jean. Éléments de Géométrie Algébrique I. Springer-Verlag, 1971.

ILLUSIE, Luc; BEILINSON, Alexander; BLOCH, Spencer; DRINFELD, Vladimir et al. Reminiscences of Grothendieck and his school. Notices of the Amer. Math. Soc., 57, (2010), p. 1106-1115.

KRONECKER, Leopold. Grundzüge einer arithmetischen theorie der algebraischen grössen. Crelle, Journal für die reine und angewandte Mathematik, XCII (1882), p. 1-122, 1882.

LANG, Serge. Algebra. 3rd ed. Reading, MASS.: Addison-Wesley, 1993.

MACLANE, Saunders. The work of Samuel Eilenberg in topology. In: HELLER, Alex; TIERNEY, Myles (ed.). Algebra, topology, and category theory: a collection of papers in honor of Samuel Eilenberg, New York: Academic Press, 1976. p. 133-144.

MCLARTY, Colin. The rising sea: Grothendieck on simplicity and generality I. In: GRAY, Jeremy; PARSHALL, Karen (eds.). Episodes in the History of Recent Algebra. American Mathematical Society, 2007. p. 301-326.

PARIKH, Carol. The unreal life of Oscar Zariski. New York: Springer-Verlag, 2009.

POINCARÉ, Henri. Analysis situs, 1895-1904. Collected in Gaston Darboux et al. (eds.). Oeuvres de Henri Poincaré in 11 volumes. Paris: Gauthier-Villars, 1916-1956. Vol. VI, p. 193-498. (Eu cito a tradução por John Stillwell, Papers on Topology: Analysis Situs and Its Five Supplements, Providence, American Mathematical Society, 2010. p. 232.

RAYNAUD, Michel. "André Weil and the foundations of algebraic geometry". Notices of the American Mathematical Society, 46 (1999), p. 864-867.

SERRE, Jean-Pierre. Faisceaux algébriques cohérents. Annals of Mathematics, 61 (1955), p. 197-277.

SERRE, Jean-Pierre. Espaces fibrés algébriques. In: Séminaire Chevalley, chapter exposé n. 1. Secrétariat Mathématique, Institut Henri Poincaré, 1958.

SERRE, Jean-Pierre. "Rapport au comitée Fields sur les travaux de A. Grothendieck (1965)". K-Theory, 3 (1989), p. 199-204.

SERRE, Jean-Pierre. "Andrée Weil". 6 May 1906-6 August 1998. Biographical Memoirs of Fellows of the Royal Society, 45 (1999), p. 520-529. 
C. McLarty - Como Grothendieck simplificou a geometria algébrica

SERRE, Jean-Pierre. Exposés de Séminaires 1950-1999. Société Mathématique de France, 2001.

SWINNERTON-DYER, Peter. A Brief Guide to Algebraic Number Theory. Cambridge University Press, 2001.

TAUSSKY, Olga. "Sums of squares". American Mathematical Monthly, 77 (1970), p. 805-830.

VAN DER WAERDEN, Bartel L. "Zur Nullstellentheorie der Polynomideale". Mathematische Annalen, 96 (1926), p. 183-208.

WEIBEL Charles. An introduction to homological algebra. Cambridge University Press, 1994.

WEIL, André. Foundations of Algebraic Geometry. American Mathematical Society, 1946.

WEIL, André. "Number of solutions of equations in finite fields". Bulletin of the American Mathematical Society, 55 (1949), p. 487-495.

WEIL, André. Number-theory and algebraic geometry. In: Proceedings of the International Congress of Mathematicians (1950: Cambridge, Mass.), p. 90-100. American Mathematical Society, 1952.

\section{Endereço postal:}

Department of Philosophy

Case Western Reserve University

10900 Euclid Ave

Cleveland, Ohio

USA 44106

Data de recebimento: 02-11-2016

Data de aceite: 07-11-2016 